Article

\title{
Driving Interactions Efficiently in a Composite Few-Body System
}

\author{
Alan Kahan ${ }^{1,2}$, Thomás Fogarty $\left.{ }^{1, *} \mathbb{(}\right)$, Jing Li $^{1}\left[\right.$ and Thomas Busch ${ }^{1}(\mathbb{D}$ \\ 1 Quantum Systems Unit, Okinawa Institute of Science and Technology Graduate University, \\ Okinawa 904-0495, Japan; kahanalan@gmail.com (A.K.); jing.li@oist.jp (J.L.); thomas.busch@oist.jp (T.B.) \\ 2 Instituto de Física Enrique Gaviola, CONICET and Universidad Nacional de Córdoba, Ciudad Universitaria, \\ Córdoba X5016LAE, Argentina \\ * Correspondence: thomas.fogarty@oist.jp
}

Received: 30 August 2019; Accepted: 1 October 2019; Published: 7 October 2019

\begin{abstract}
We study how to efficiently control an interacting few-body system consisting of three harmonically trapped bosons. Specifically, we investigate the process of modulating the inter-particle interactions to drive an initially non-interacting state to a strongly interacting one, which is an eigenstate of a chosen Hamiltonian. We also show that for unbalanced subsystems, where one can individually control the different inter- and intra-species interactions, complex dynamics originate when the symmetry of the ground state is broken by phase separation. However, as driving the dynamics too quickly can result in unwanted excitations of the final state, we optimize the driven processes using shortcuts to adiabaticity, which are designed to reduce these excitations at the end of the interaction ramp, ensuring that the target eigenstate is reached.
\end{abstract}

Keywords: shortcuts to adiabaticity; cold atoms; few-body systems

\section{Introduction}

The ability to precisely control quantum systems is a prerequisite for developing technologies in the areas of quantum computation, simulation, and metrology. Even though the control over single-particle states is highly developed by today [1-3], the requirements stemming from short decoherence time-scales are often hard to fulfill. Even more so, fast operations can have detrimental effects on quantum states, as possible imperfections in the control pulses can be difficult to compensate. To mitigate these problems, and to ensure high fidelity on short timescales, a number of techniques have been developed, such as optimal control algorithms [4-6] and shortcuts to adiabaticity (STAs) [7-11]. In this work we will focus on STAs, which are techniques designed to determine the driving parameters such that the system undergoes adiabatic evolution within a finite time, and which have been successfully employed in recent experiments with cold atoms [12-17]. While for single particles or within a mean-field approximation, the description of the exact evolution of the quantum state is tractable, extending such a treatment to interacting many-body systems poses complications due to their complexity. One solution is to use approximate variational techniques, and even though they are not exact, use these to design STA processes [18-20]. In fact, it has recently been shown that such an approach can be used to control correlations in small systems [21] and can serve as a good benchmark for the control of larger interacting systems.

In this work, we aim to move beyond mean-field and single-particle physics to control the dynamics of larger systems with short-range contact interactions. To achieve this, we take a first step by focusing on nontrivial few-body states, in particular, controlling the interactions in a bosonic two-component system confined to a harmonic trap. Such systems can exhibit complex dynamics arising from the interplay of intra- and inter-species interactions, leading, for example, to composite 
fermionization or phase separation [22-25]. To discuss the basic effects, we will focus on a paradigmatic realization of a two-component system, namely, two interacting ultracold atoms of species A, which interacts with a single ultracold atom of species B in a one-dimensional setting. In such a system, the interactions can be described by point-like potentials, and in ultracold atom experiments, the interaction strength can be changed by employing Feshbach [26,27] or confinement-induced resonances [28]. In fact, individual tuning of the inter- and intra-species interactions allows one to explore driving the interactions of the A atoms in the presence of an impurity atom, driving the interaction between an impurity and the interacting $\mathrm{A}$ atoms, and also driving all interactions simultaneously. With this freedom, it is possible to explore driving the system through the phase separation transition, which can alter the ordering of the particles in the trap and, therefore, greatly affect the dynamics of the system. We will show that efficient STAs can be designed for the individual interactions based on a variational ansatz, and that these STAs can outperform a non-optimized interaction ramp for most timescales of the driven process.

In Section 2, we introduce the few-body model we consider, and in Section 3, we describe the variational method for designing STAs in this system. We begin in Section 4 with the analysis of driving interactions between three identical bosons, while in Sections 5 and 6, we investigate driving one of the interaction terms while the other is held fixed. These latter sections describe the effect phase separation has on the driven system and its dynamics. Finally, we conclude.

\section{Model}

We consider a one-dimensional system of three interacting bosons of mass $m$, confined in a harmonic trap of frequency $\omega$, whose Hamiltonian is given by

$$
H=\sum_{j=1}^{3}\left[-\frac{\hbar^{2}}{2 m} \nabla_{j}^{2}+\frac{1}{2} m \omega^{2} x_{j}^{2}\right]+V_{\mathrm{int}}\left(x_{1}, x_{2}, x_{3}\right) .
$$

At low temperatures, we can assume that the scattering between the particles is mostly two-body and of the s-wave form, allowing us to approximate the interaction part of the Hamiltonian with point-like pseudo-potentials as

$$
V_{\text {int }}\left(x_{1}, x_{2}, x_{3}\right) \approx g^{A}(t) \delta\left(x_{1}-x_{2}\right)+g^{A B}(t)\left[\delta\left(x_{1}-x_{3}\right)+\delta\left(x_{2}-x_{3}\right)\right] .
$$

Here, the interaction strength between two particles of species A is given by $g^{A}$, and the interaction strength between an A particle and the $\mathrm{B}$ particle is given by $g^{A B}$. Both couplings are related to the respective $3 \mathrm{D}$ scattering lengths, $a_{3 D}$, via $g=\frac{4 \hbar^{2} a_{3 D}}{m d_{\perp}^{2}} \frac{1}{1-C_{3 D} \frac{a_{\perp}}{d_{\perp}}}$, with the constant $C$ given by $C \approx$ 1.4603 [28]. Here, $\omega_{\perp}$ is the trap frequency in the transverse directions of a quasi-one-dimensional harmonic trap of width $d_{\perp}=\sqrt{\hbar / m \omega_{\perp}}$, and one can see that control over the transverse trap frequency or the scattering length allows one to tune the interaction strengths. In the following, we will use harmonic oscillator units by rescaling all spatial coordinates with $a_{0} \equiv \sqrt{\frac{\hbar}{m \omega}}$, all interaction strengths in units of $\sqrt{2} a_{0} \hbar \omega$, all energies in units of $\hbar \omega$, and time in units of $\omega^{-1}$.

The Hamiltonian given in (1) can be separated by introducing Jacobi coordinates

$$
\begin{aligned}
& X=\left(x_{1}-x_{2}\right) / \sqrt{2}, \\
& Y=\left(x_{1}+x_{2}\right) / \sqrt{6}-\sqrt{2 / 3} x_{3}, \\
& Z=\left(x_{1}+x_{2}+x_{3}\right) / \sqrt{3},
\end{aligned}
$$


where $X$ and $Y$ are two relative coordinates describing the positions of the three particles with respect to each other, while $Z$ describes the system's center-of-mass. The latter separates, and in these new coordinates, the Hamiltonian can be written as $H=H_{\text {com }}(Z)+H_{\text {rel }}(X, Y)$, with

$$
\begin{aligned}
H_{\mathrm{com}} & =-\nabla_{Z}^{2}+\frac{1}{2} Z^{2}, \\
H_{\mathrm{rel}} & =-\frac{1}{2}\left(\nabla_{X}^{2}+\nabla_{Y}^{2}\right)+\frac{1}{2}\left(X^{2}+Y^{2}\right)+g^{A} \delta(X)+g^{A B}\left[\delta\left(-\frac{1}{2} X+\frac{\sqrt{3}}{2} Y\right)+\delta\left(-\frac{1}{2} X-\frac{\sqrt{3}}{2} Y\right)\right] .
\end{aligned}
$$

It is immediately clear that the center-of-mass part just describes a particle moving in a harmonic trap, for which the solutions are given by:

$$
\psi_{n}^{H O}(Z)=\pi^{-1 / 4}\left(2^{n} n !\right) H_{n}(Z) \exp \left(-Z^{2} / 2\right),
$$

where $H_{n}(Z)$ are the Hermite polynomials, and with energies $E_{n}=(n+1 / 2)$. The relative Hamiltonian can be interpreted as describing the effectively two-dimensional motion of a harmonically trapped particle in the presence of three narrow barriers arranged with a $\pi / 3$ angle between them, as depicted in Figure 1a. Since the two A atoms are identical, the wave-function has to remain unchanged under a reflection across the $X$ coordinate; however, there is no constraint when swapping particle B with one of the A particles. Exact solutions exist for limiting cases of the interactions [29,30], while in general, this system can be solved effectively using exact diagonalization techniques [24,31,32]. Indeed, for $g^{A} \neq g^{A B}$, phase separation can be observed, whereby either the particles of species $A$ are pushed to the edges of the trap, while particle B is confined in the trap center, or vice versa [23,32-35]. Therefore, the arrangement of the atoms in the trap is non-trivial and will be strongly affected by any change in their inter- or intra-species interactions. It makes this an ideal system in which to study all regimes of composite few-body dynamics and to develop useful quantum control techniques.

In the following, we will concentrate on increasing the interaction strength in initially non-interacting and uncorrelated systems on time scales $t_{f}$, which are approximately comparable to the trap period. As the center-of-mass contribution does not depend on the interaction, it will be unchanged during the interaction ramping process, and therefore, only the dynamics of the relative wave-function $i \hbar \frac{\partial}{\partial t} \psi(X, Y ; t)=H_{\text {rel }}(t) \psi(X, Y ; t)$ need to be considered.

For very slow ramps of the interaction $\left(t_{f} \ggg 1\right)$, the dynamics can be considered to be adiabatic, and the energy of the state after the process is equal to the energy of the eigenstate at the final value of the interaction strength, $g_{f}$, i.e., $E^{A D} \equiv E\left(t_{f}\right)=E\left(g_{f}\right)$. For faster ramps $\left(t_{f} \sim \mathcal{O}(1)\right)$, the process can be non-quasi-static, resulting in excess energy in the system due to the out-of-equilibrium dynamics, with non-adiabatic energy $E^{N A}\left(t_{f}\right) \geq E^{A D}$. We can then define irreversible work from this non-adiabatic energy as

$$
\left\langle W_{i r r}\right\rangle=E^{N A}\left(t_{f}\right)-E^{A D},
$$

which for adiabatic processes will vanish, while being finite for non-quasi-static processes [21,36]. It is therefore a useful quantity to characterize the efficiency of the interaction ramp.

In the following, we will describe two kinds of interaction ramps. The first will be a generic, non-optimized reference ramp, which we will use as a benchmark and which we parameterize as

$$
g^{\mathrm{ref}}(t)=\frac{g_{f}}{32}\left[30 \sin \left(\frac{\pi}{2} \frac{t}{t_{f}}\right)-5 \sin \left(\frac{3 \pi}{2} \frac{t}{t_{f}}\right)-3 \sin \left(\frac{5 \pi}{2} \frac{t}{t_{f}}\right)\right] .
$$

This function satisfies the boundary conditions $g^{\text {ref }}(0)=0, g^{\text {ref }}\left(t_{f}\right)=g_{f}$, and $\dot{g}^{\text {ref }}(0)=\dot{g}^{\text {ref }}\left(t_{f}\right)=$ $\ddot{g}^{\text {ref }}(0)=\ddot{g}^{\text {ref }}\left(t_{f}\right)=0$, ensuring that the interaction ramp begins and ends smoothly in an effort to reduce untypical excitations. However, this assumption alone will not ensure that no irreversible 
dynamics are created during the ramping processes, and the pulse can therefore serve as a reference to an optimized interaction ramp derived from the STA approach. This comparison will be our main tool to quantify the success of the designed STA, which we will develop in the next section.

\section{Shortcut to Adiabaticity}

To find an STA, we use the method of inverse engineering, which can design interaction ramps $g^{S T A}(t)$ that fulfill the desired adiabatic evolution of the system $\phi(X, Y ; t)$ for any ramp time $t_{f}$. The success of the STA then depends entirely on how well the solutions to the time-dependent Hamiltonian, $\phi(X, Y ; t)$, are known and whether they possess scale invariance [37]. As our interaction ramp is not scale invariant and since exact forms of the solution are not known, one has to use approximate techniques $[19,38,39]$, and we therefore employ a variational approach with an ansatz that describes the evolution of the relative wave-function by an interpolation between the initial and the final state

$$
\begin{aligned}
\phi(X, Y ; t) & =\varphi(X, Y ; t) e^{i\left(b(t) X^{2}+c(t) Y^{2}\right)} \\
& =N(t)\left[(1-\eta(t)) \phi_{i}(X, Y)+\eta(t) \phi_{f}(X, Y)\right] e^{i\left(b(t) X^{2}+c(t) Y^{2}\right)}
\end{aligned}
$$

Here, $N(t)$ is a time-dependent normalization constant, while $b(t)$ and $c(t)$ are chirps that allow the wave-function to change its width. To ensure that the wave-function changes smoothly from the initial state $\phi_{i}(X, Y) \equiv \phi\left(X, Y ; g_{i}\right)$ to the target state $\phi_{f}(X, Y) \equiv \phi\left(X, Y ; g_{f}\right)$, we choose $\eta(t)$ as a $6^{\text {th }}$ order polynomial satisfying the boundary conditions $\eta(0)=0, \eta\left(t_{f}\right)=1$ and $\dot{\eta}(0)=\ddot{\eta}(0)=\dot{\eta}\left(t_{f}\right)=$ $\ddot{\eta}\left(t_{f}\right)=0$.

(a)

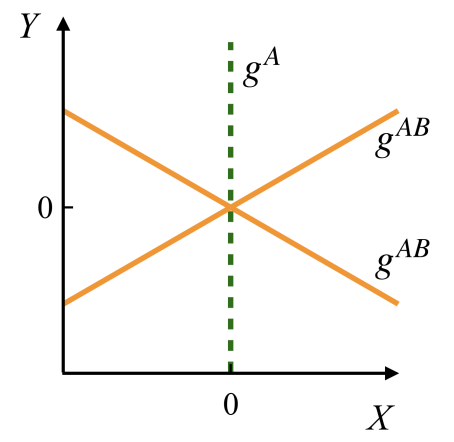

(b)

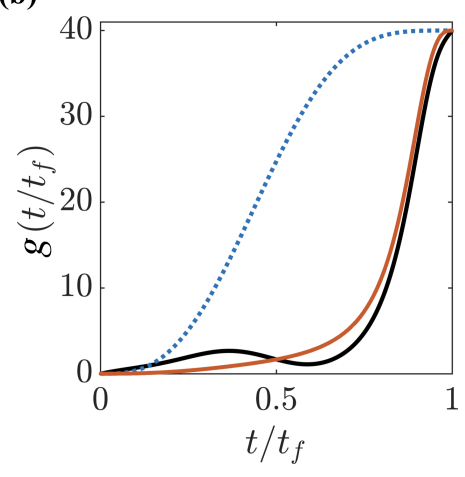

Figure 1. (a) Interaction potentials stemming from $g^{A}$ (dashed line) and $g^{A B}$ (solid lines) in the Jacobi coordinate plane. (b) Examples of different interaction ramps when keeping $g^{A}(t)=g^{A B}(t)$, with the reference ramp (blue dotted line) and the shortcut to adiabaticity (STA) ramp at $t_{f}=1.5$ (black solid line) and $t_{f}=10$ (red solid line).

The next step in the process is to minimize the action of the effective Lagrangian [18]

$$
\mathcal{L}=\int_{-\infty}^{\infty} d X \int_{-\infty}^{\infty} d Y\left[\frac{i}{2}\left(\frac{\partial \phi}{\partial t} \phi^{*}-\frac{\partial \phi^{*}}{\partial t} \phi\right)-\frac{1}{2}\left|\frac{\partial \phi}{\partial X}\right|^{2}-\frac{1}{2}\left|\frac{\partial \phi}{\partial Y}\right|^{2}-V(X, Y ; t)|\phi|^{2}\right]
$$

where $V=\frac{1}{2}\left(X^{2}+Y^{2}\right)+g^{A}(t) \delta(X)+g^{A B}(t)\left[\delta\left(-\frac{1}{2} X+\frac{\sqrt{3}}{2} Y\right)+\delta\left(-\frac{1}{2} X-\frac{\sqrt{3}}{2} Y\right)\right]$ contains the trapping and the interaction potentials. This effective Lagrangian is minimized with respect to the variational parameters $\eta(t), b(t)$, and $c(t)$, and the resulting Euler-Lagrange equations give the 
evolution equations that will determine $b(t)$ and $c(t)$ and establish the time dependence of the two interaction strengths, $g^{A}(t)$ and $g^{A B}(t)$, given by

$$
g^{A}(t) I_{A}+g^{A B}(t) I_{A B}=-\left[\frac{\partial \xi^{2}}{\partial \eta}\left(\dot{b}+2 b^{2}+\frac{1}{2}\right)+\frac{\partial v^{2}}{\partial \eta}\left(\dot{c}+2 c^{2}+\frac{1}{2}\right)+\frac{1}{2} \frac{\partial}{\partial \eta}(\beta+\gamma)\right] .
$$

Here, $\xi^{2}=\int_{\infty}^{\infty} d X \int_{\infty}^{\infty} d Y X^{2}|\varphi|^{2}$ and $v^{2}=\int_{\infty}^{\infty} d X \int_{\infty}^{\infty} d Y Y^{2}|\varphi|^{2}$ are the widths of the state in the $X$ and $Y$ directions, respectively, and their dynamics is determined by

$$
\begin{aligned}
& \dot{\xi}=2 b \xi, \\
& \dot{v}=2 c v .
\end{aligned}
$$

Similarly, the kinetic energies in these directions are $\beta=\int_{\infty}^{\infty} d X \int_{\infty}^{\infty} d Y\left|\frac{\partial \varphi}{\partial X}\right|^{2}$ and $\gamma=$ $\int_{\infty}^{\infty} d X \int_{\infty}^{\infty} d Y\left|\frac{\partial \varphi}{\partial Y}\right|^{2}$, and the interaction energies are $I_{A}=\int_{\infty}^{\infty} d X \int_{\infty}^{\infty} d Y \varphi \delta(X)$ and $I_{A B}=$ $\int_{\infty}^{\infty} d X \int_{\infty}^{\infty} d Y \varphi\left[\delta\left(-\frac{X}{2}+\frac{\sqrt{3}}{2} Y\right)+\delta\left(-\frac{X}{2}-\frac{\sqrt{3}}{2} Y\right)\right]$. Using known solutions to $\phi_{i}$ and $\phi_{f}$ allows us to calculate these terms exactly (for example, in the limit of infinite repulsive interactions, accurate approximations are known [29,30]); otherwise, these integrals can be calculated numerically. Typical examples of these STA interaction ramps for a system of three identical particles are shown in Figure $1 \mathrm{~b}$ for two timescales $t_{f}=\{1.5,10\}$. When $t_{f}$ is large, the STA is designed to quickly ramp the interaction at the end of the process, in contrast to the reference, which begins to slowly increase the interaction already at the beginning of the ramp. This difference is due to the optimization of the STA through the Lagrangian, which is designed taking into account the energy dependence on the interaction and is similar to that seen in smaller systems [21]. For small $t_{f}$, the STA possesses large modulations as driving the system faster requires large changes in the energy to follow the adiabatic path.

\section{Three Identical Particles}

Assuming that the initial state is the ground state of the noninteracting system, $g_{i}=g_{i}^{A}=g_{i}^{A B}=0$ (see Figure 2a), we investigate in the following the ramping of strong interactions between three identical particles, i.e., $g(t)=g^{A}(t)=g^{A B}(t)$. In this case, the relative part of the wave-function will always possess $C_{6 v}$ symmetry $[31,40,41]$ (see Figure $2 b-d$ ), with the interactions leading to cusps in the density at $60^{\circ}$ angles to each other, and with the cusp asymptotically reaching zero density in the Tonks-Girardeau limit of strong repulsive interactions $\left(g_{f} \gtrsim 40\right)$ [42-44].

To quantify the success of the interaction ramps, we compare the irreversible work $\left\langle W_{i r r}\right\rangle$ after the respective interaction ramps (see Figure 2e). For longer ramp times, $t_{f}>25$, the system is driven slowly enough that it can be considered as evolving adiabatically, which results in a vanishing $\left\langle W_{i r r}\right\rangle$ and therefore, a high-fidelity process for any reasonable non-optimized reference ramp. However, for short ramp times, $t_{f} \lesssim 10$, ramping to stronger interactions creates more irreversible dynamics as the system is driven further from its equilibrium, resulting in large $\left\langle W_{i r r}\right\rangle$ and therefore, low-fidelity final states. It is on these timescales that we see the advantages of using the STA as it outperforms the non-optimized reference ramp, possessing lower amounts of $\left\langle W_{i r r}\right\rangle$ for the different final interactions. The modulations visible in $\left\langle W_{i r r}\right\rangle$ for the STA are due to excitations of the system to high energy states that possess the same symmetry as the ground state. While the contribution of these excitations is small for long ramp times, when driving the system quickly the STA is unsuccessful in damping them due to its approximate form through the ansatz in Equation (11). Indeed, divergence of $\left\langle W_{i r r}\right\rangle$ occurs for timescales $t_{f} \lesssim 1$, when the STA ramp becomes negative at certain time intervals and destabilizes the system. This sets a limitation on the operation of our STA to times $t_{f}>1$. 


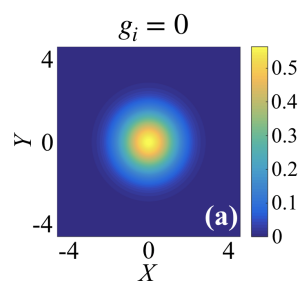

$g_{f}=1$

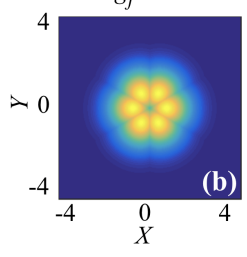

$g_{f}=40$

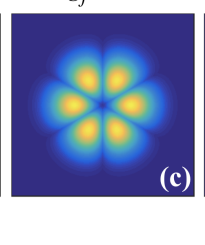

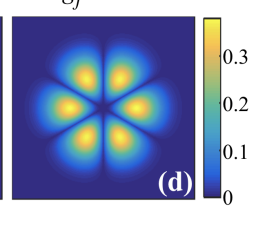

(e)

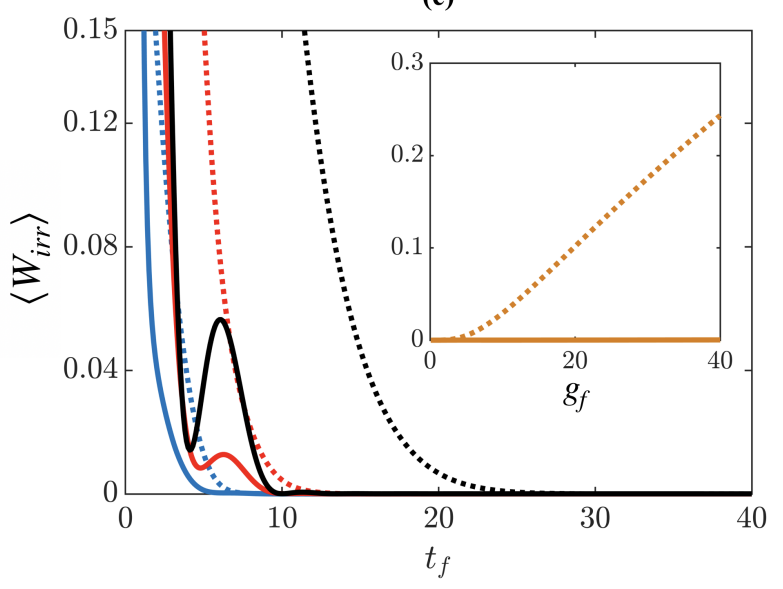

Figure 2. (a) Initial state in the relative $\{X, Y\}$ coordinate plane. Target states at (b) $g_{f}=1,(\mathbf{c}) g_{f}=5$, and (d) $g_{f}=40$. (e) $\left\langle W_{i r r}\right\rangle$ for three indistinguishable particles as a function of the ramp time $t_{f}$ for the STA (solid lines) and reference ramp (dotted lines). The final interactions are $g_{f}=1$ (blue lines), $g_{f}=5$ (red lines), and $g_{f}=40$ (black lines). Inset shows $\left\langle W_{i r r}\right\rangle$ versus final interaction strength $g_{f}$ at $t_{f}=10$.

While for ramps to weak interactions the STA and the reference give comparable results, the difference between them increases when driving to larger interactions (see inset of Figure 2e). When using the STA, the final state is essentially reached with $t_{f}=10$ for any final interaction; however, applying the reference ramp drives the system further from the target eigenstate with growing interactions. This is not surprising as driving quickly to an infinitely repulsive state results in a diverging energy expectation value [45,46]; however, in this case the STA allows us to drive the system significantly faster than the reference as the excitations are successfully suppressed.

Finally, we compare the structure of the three-body state through comparisons of the one-body density matrix (OBDM), whereby we examine the reduced state after tracing out two particles from the system

$$
\begin{aligned}
\rho^{A}\left(x_{1}, x_{1}^{\prime}\right) & =\int_{-\infty}^{+\infty} \Psi\left(x_{1}, x_{2}, x_{3}\right) \Psi^{*}\left(x_{1}^{\prime}, x_{2}, x_{3}\right) d x_{2} d x_{3} \\
\rho^{B}\left(x_{3}, x_{3}^{\prime}\right) & =\int_{-\infty}^{+\infty} \Psi\left(x_{1}, x_{2}, x_{3}\right) \Psi^{*}\left(x_{1}, x_{2}, x_{3}^{\prime}\right) d x_{1} d x_{2} .
\end{aligned}
$$

Here, $\rho^{A}\left(x_{1}, x_{1}^{\prime}\right)$ is the OBDM of a particle of species A after tracing out the other A particle and the particle $\mathrm{B}$, while $\rho^{B}\left(x_{3}, x_{3}^{\prime}\right)$ is the OBDM of particle $\mathrm{B}$ after tracing out the two A particles. In the case when $g^{A}=g^{A B}$, both of these reduced states are equivalent; however, this is not necessarily true when $g^{A} \neq g^{A B}$, as the components will be distinguishable and the rotational symmetry of the ground will be broken.

In Figure 3, we compare the OBDM after the STA and reference interaction ramps (at $t_{f}=10$ ) with that of the target OBDM. While ramps to weakly interacting states $\left((\mathrm{a}, \mathrm{d}) g_{f}=1\right.$ and $(\mathrm{b}, \mathrm{e})$ $g_{f}=5$ ) yield exactly the same OBDM after either of the ramps, the results are different when driving to strong interactions $\left((\mathrm{c}, \mathrm{f}) g_{f}=40\right)$. Indeed, the OBDM after the STA is equivalent to the target OBDM; however, the OBDM after the reference is markedly different. Here it is instructive to compare the diagonal density $\left(\rho\left(x=x^{\prime}\right)\right)$ of the reference OBDM to the target OBDM, which indicates that it has a broader width. In fact, each particle localizes spatially when the interactions are strongly repulsive, resulting in three density modulations about the center of the trap; however, the reference interaction ramp has imparted large kinetic energy to the system, pushing the particles further from their equilibrium positions. In comparison, the STA has precisely modulated the interaction to ensure that any non-adiabatic energy is removed from the system by the end of the ramp, resulting in a final state that is an eigenstate of the Hamiltonian. 

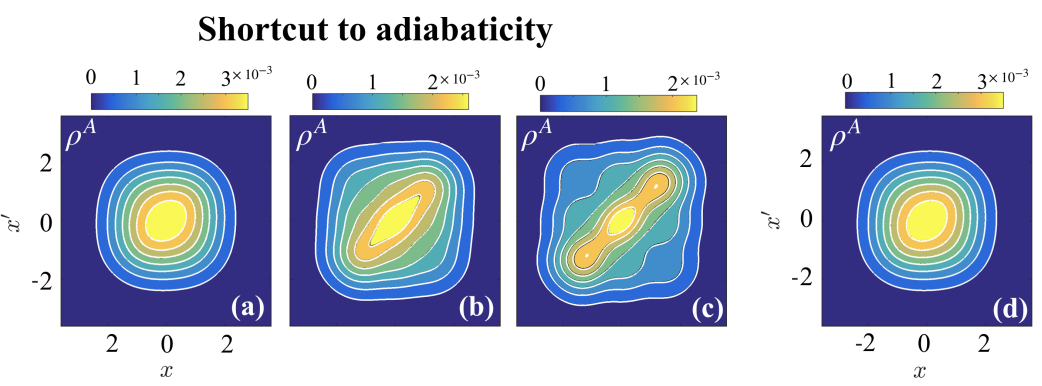

\section{Reference}
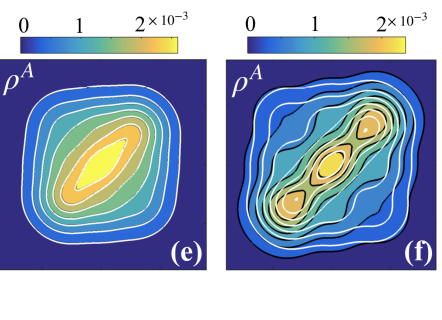

Figure 3. Target one-body density matrices (OBDMs, (white contour lines) on top of final OBDMs for three identical particles at $t_{f}=10$. Panels $(\mathbf{a}-\mathbf{c})$ correspond to the STA, while panels $(\mathbf{d}-\mathbf{f})$ correspond to the reference pulse. Panels (a) and (d) are for $g_{f}=1,(\mathbf{b})$ and (e) for $g_{f}=5$, and (c) and (f) for $g_{f}=40$.

\section{Driving in the Presence of Weak Fixed Interactions}

Let us now consider that the inter- and intra-species interactions are different, such that we drive one interaction term while the other interaction term is held fixed at a low value. Then, due to the ability to tune both the inter- and intra-species interactions separately, one can consider two different setups. The first allows the tuning of the interactions between the A particles whilst in the presence of the impurity B atom, which we will refer to as system driving. At the same time, the inter-species interaction between the impurity and the two $\mathrm{A}$ atoms is fixed at $g^{A B}=1$, such that the initial state at $g_{i}^{A}=0$ possesses small cusps along the directions $Y= \pm X / \sqrt{3}$ (see Figure 4a). Driving the interaction $g^{A}(t)>0$ between the A atoms will introduce a delta-function interaction potential that bisects the $X$-axis and forces squeezing of the density from the $X$ to the $Y$ direction (see target states in panels (b) to (d) in Figure 4).
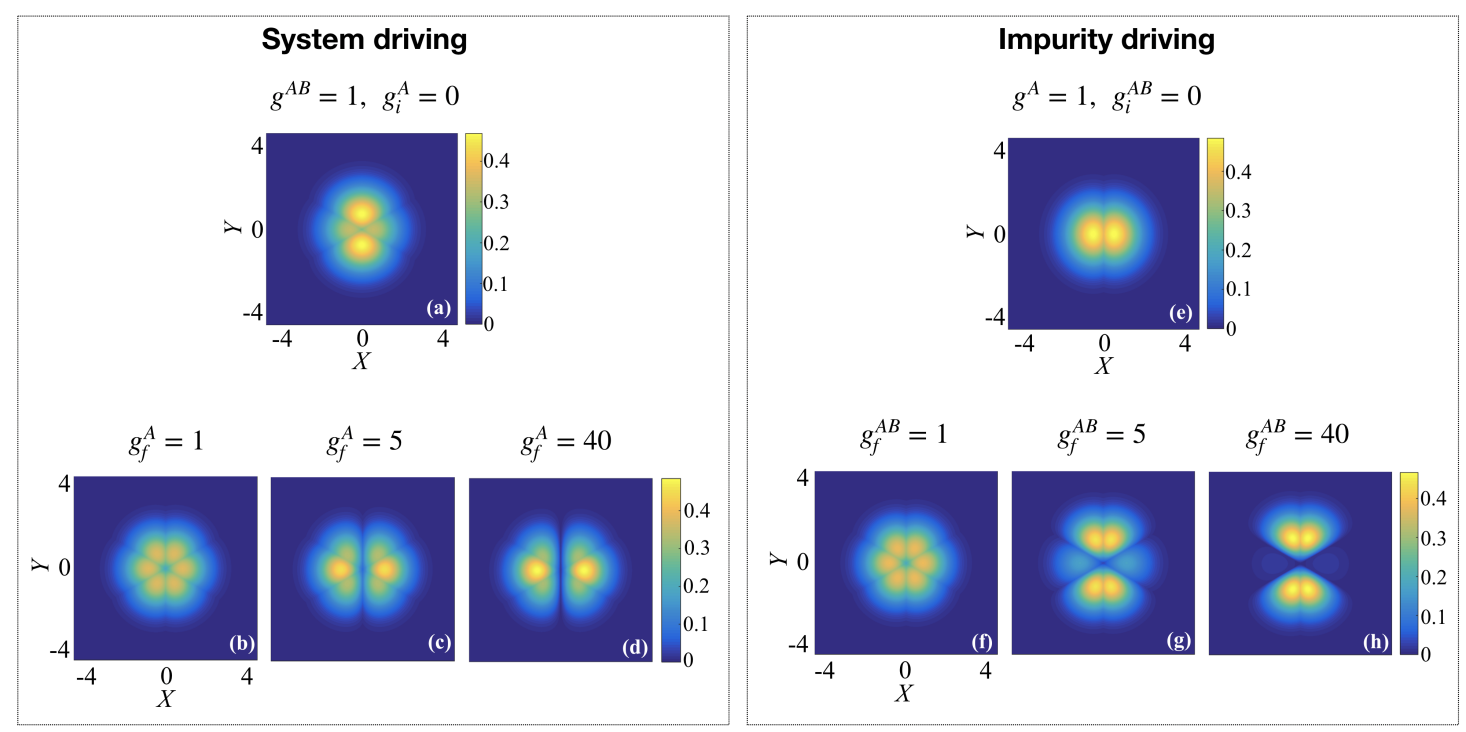

Figure 4. (a) Initial state with $g^{A B}=1$ and $g_{i}^{A}=0$ and (b-d) target states for $g_{f}^{A}=\{1,5,40\}$. This case is referred to as system driving. (e) Initial state with $g^{A}=1$ and $g_{i}^{A B}=0$ and (f-h) target states for $g_{f}^{A B}=\{1,5,40\}$. This case is referred to as impurity driving.

The second setup allows switching on the interaction with an impurity atom in a weakly interacting two-body system, which we will refer to as impurity driving. For this, we consider the intra-species interaction to always be fixed at $g^{A}=1$, while the interaction with the $\mathrm{B}$ atom is initially $g_{i}^{A B}=0$. The initial state therefore describes two weakly interacting A bosons, with the interaction bisecting the $X$-axis of the relative part of the wave-function (see Figure 4e). Driving the interactions $g^{A B}(t)>0$ between the impurity and the $\mathrm{A}$ atoms will introduce delta-function interaction potentials along $Y= \pm X / \sqrt{3}$ and force the density to be squeezed in the $X$ direction when 
the fixed interaction between the A particles is weaker than the impurity interactions (see target states in panels (g) and (h) in Figure 4).

We assess the effect of the system driving dynamics through the irreversible work as shown in Figure $5 \mathrm{a}$. One can clearly see that for ramp times $t_{f} \geq 10$, the STA dynamics lead to the ground state irregardless of the strength of the final interaction $g_{f}^{A}$, while the reference ramp leads to increasing $\left\langle W_{i r r}\right\rangle$ for stronger interactions on the same time scale (see inset in panel (a)). However, for times $t_{f}<10$, the STA becomes less effective, similar to the case of the simultaneous driving of $g^{A}$ and $g^{A B}$ discussed in Section 4 and for the results found in [21].

(a)

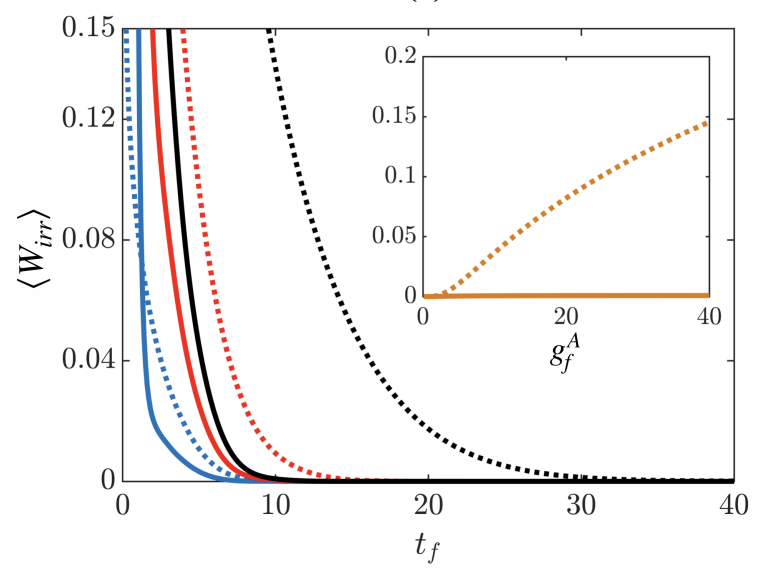

(b)

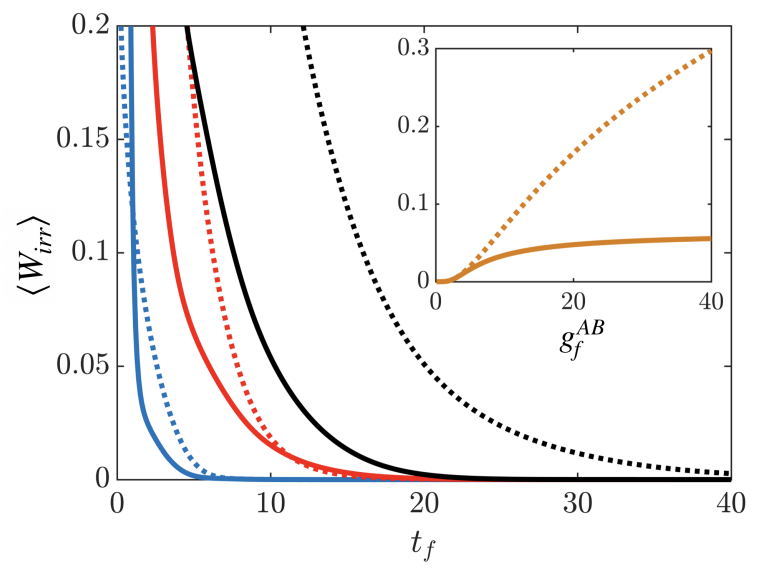

Figure 5. (a) $\left\langle W_{i r r}\right\rangle$ after driving the system interactions in the presence of a weak fixed impurity interaction $g^{A B}=1$. System interactions are driven to $g_{f}^{A}=1$ (blue lines), $g_{f}^{A}=5$ (red lines), and $g_{f}^{A}=40$ (black lines), with the solid lines showing the result of the STA and the dotted lines showing the result of the reference ramp. (b) Impurity driving in the presence of weak fixed system interactions $g_{A}=1$, with final impurity interactions $g_{f}^{A B}=1$ (blue lines), $g_{f}^{A B}=5$ (red lines), and $g_{f}^{A B}=40$ (black lines). Insets show $\left\langle W_{\text {irr }}\right\rangle$ as a function of $g_{f}^{A}$ and $g_{f}^{A B}$, respectively, at $t_{f}=10$.

For the case of driving the impurity, we show $\left\langle W_{i r r}\right\rangle$ in Figure $5 \mathrm{~b}$. The results here are subtly different to the previous case as the STA seemingly gives less of an advantage over the reference ramp when the interactions are weak, $g_{f}^{A B} \leq 5$. In fact, adiabaticity (when $\left\langle W_{i r r}\right\rangle \rightarrow 0$ ) is reached for similar timescales for both the reference and the STA, and only when driving to large interactions, $g_{f}^{A B}=40$, the STA performs significantly better (see inset in panel (b)).

The fact that driving the system and driving the impurity produce qualitatively different results with regards to the creation of irreversible work can be explained by the need for a spatial re-organization of the particles during the interaction ramp, which can be observed in the structure of the OBDMs for the system and the impurity (see Figure 6). Firstly, driving the system interactions increases the repulsion between the two A particles, resulting in the appearance of two maxima in the diagonal density for the $A$ particles (see panels $(b-1, c-1)$ ), while the OBDM of the impurity $B$ atom is relatively unchanged due to the weak inter-species interaction $g^{A B}=1$ (see panels (b-2,c-2)). Similar to Section 4 , the STA successfully reaches the target eigenstate, while the reference forces the two A atoms further apart due to its inefficient driving (see (e-1,f-1)). In comparison, driving the impurity interaction forces the $B$ atom to split and occupy the trap edges, being in a superposition of the left and right sides of the trap (see panels (h-2,i-2)). The strong inter-species interaction squeezes the density of the A atoms as they sit in the middle of the trap surrounded by the B particle (panels (h-1,i-1)). In this case, the splitting of the impurity atom is a manifestation of phase separation in microscopic systems as the lighter species moves to the trap edges $[23,32,34,35]$. Driving to a phase separated state is known to lead to large irreversible dynamics as the particles oscillate between the miscible and immiscible regimes [47-49]. Therefore, the STA is less effective at driving the system quickly; however, it can still 
outperform the non-optimized reference ramp (see panels $(\mathrm{k})$ and $(\mathrm{l})$ ), bringing the final state closer to the target eigenstate.
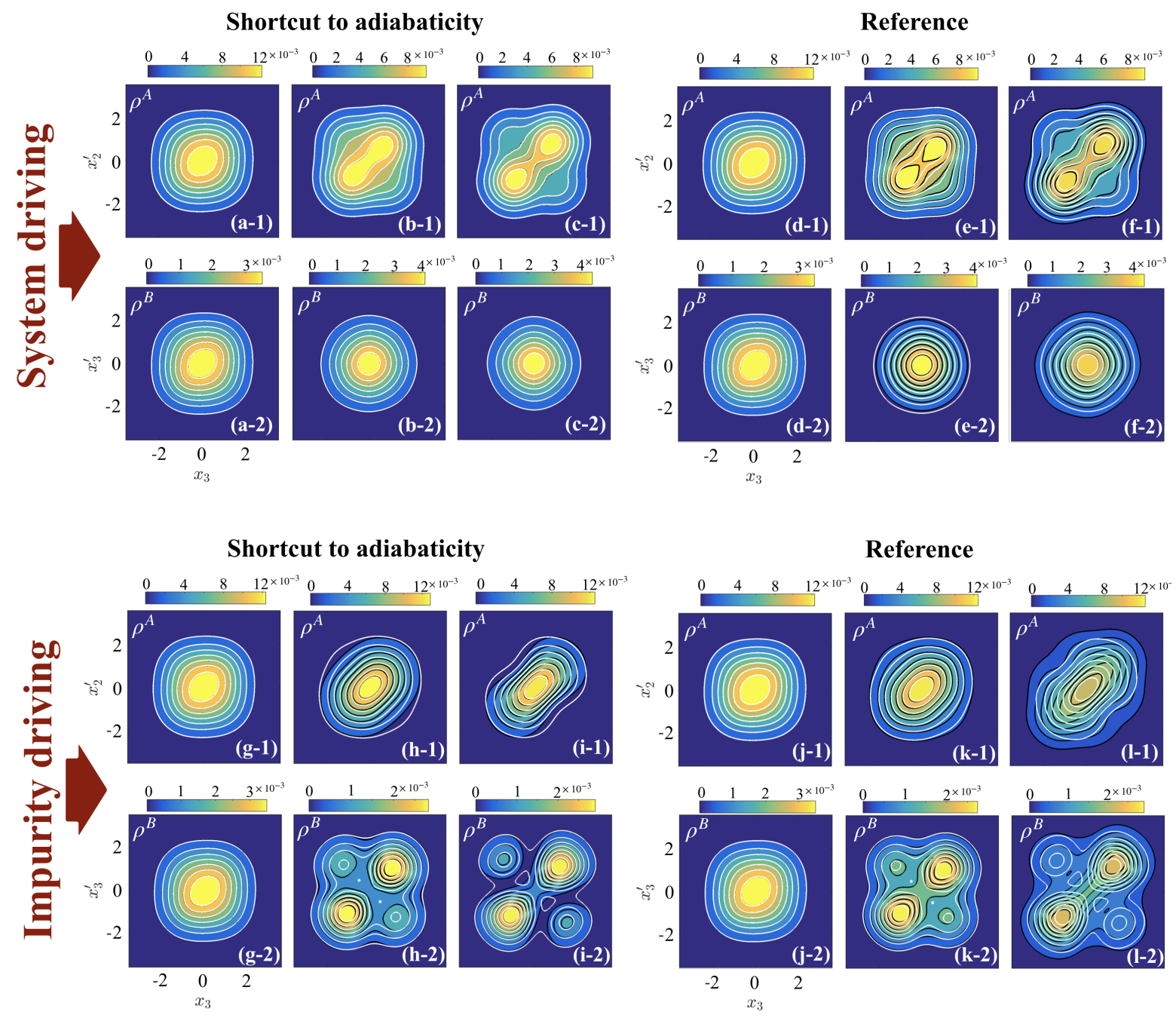

Figure 6. Panels (a-f): Target states (white contour lines) on top of final states (at $t_{f}=10$ ) for driving system interactions between $\mathrm{A}$ atoms in the presence of a fixed impurity interaction $g^{A B}=1$. Panels with index $(-1)$ correspond to $\rho^{A}\left(x_{1}, x_{1}^{\prime}\right)=\rho^{A}\left(x_{2}, x_{2}^{\prime}\right)$ while panels with index $(-2)$ show $\rho^{B}\left(x_{3}, x_{3}^{\prime}\right)$. Panels (a-c) show the STA final states while panels (d-f) show the final states for the reference pulse, where panels (a) and (d) are for $g_{f}^{A}=1,(\mathbf{b})$ and (e) are for $g_{f}^{A}=5$, and (c) and (f) are for $g_{f}^{A}=40$. Panels (g-l): Target states (white contour lines) on top of final states (at $t_{f}=10$ ) for driving impurity interactions in the presence of a fixed interactions between the A atoms $g^{A}=1$. Panels $(\mathbf{g})$ and $(\mathbf{j})$ are for $g_{f}^{A B}=1,(\mathbf{h})$ and $(\mathbf{k})$ are for $g_{f}^{A B}=5$, and (i) and (1) are for $g_{f}^{A B}=40$.

\section{Driving in the Presence of Strong Fixed Interactions}

Let us next consider the driving of one interaction term while the other interaction term is held fixed at a large value, which we can again separate into two different setups. Driving the system interactions $g^{A}(t)$ when the impurity interaction is fixed at $g^{A B}=20$, and driving the impurity interactions $g^{A B}(t)$ when the system interactions are fixed at $g^{A}=20$. Similar to the previous section, the presence of strong interactions in this system can result in the formation of different structural phases as the individual interaction terms are ramped. For instance, in the case of system driving, a strong interaction with the impurity at $t=0$ forces the relative wave-function density to align along the $X=0$ axis while being suppressed along the $Y=0$ axis (see Figure $7 \mathrm{a}$ ). Increasing the interactions 
among the A atoms then drives them apart along the $X$ direction (see panels (b) and (c)) and forces the density to redistribute to the previously unoccupied sectors between dips caused by the $g^{A B}$ interactions. At $g_{f}^{A}=40$, the orientation of the maximum density is rotated by $90^{\circ}$ from the initial state and lies along the $Y=0$ axis (see panel (d)). The inverse of this process is observed when driving the impurity interaction in the presence of strong $g^{A}$ (see panels $(\mathrm{e}-\mathrm{h})$ ). This reorganization of the particle density highlights the emergence of different phase-separated regimes in the three-particle system. Specifically, for fixed impurity interactions $g^{A B}=20$ and for a final system interaction of $g_{f}^{A}=5$, the A particles are surrounded by the B particle (in the configuration BAB), while for $g_{f}^{A}=40$ the orientation is flipped, with the B particle surrounded by the A particles (in the configuration ABA). Similarly, for fixed system interaction $g^{A}=20$ and $g_{f}^{A B}=5$, the B particle is surrounded by the A particles (ABA), and when $g_{f}^{A B}=40$, the A particles are surrounded by the B particle (BAB). Transitioning between the different phase-separated regimes will become important when driving the dynamics.
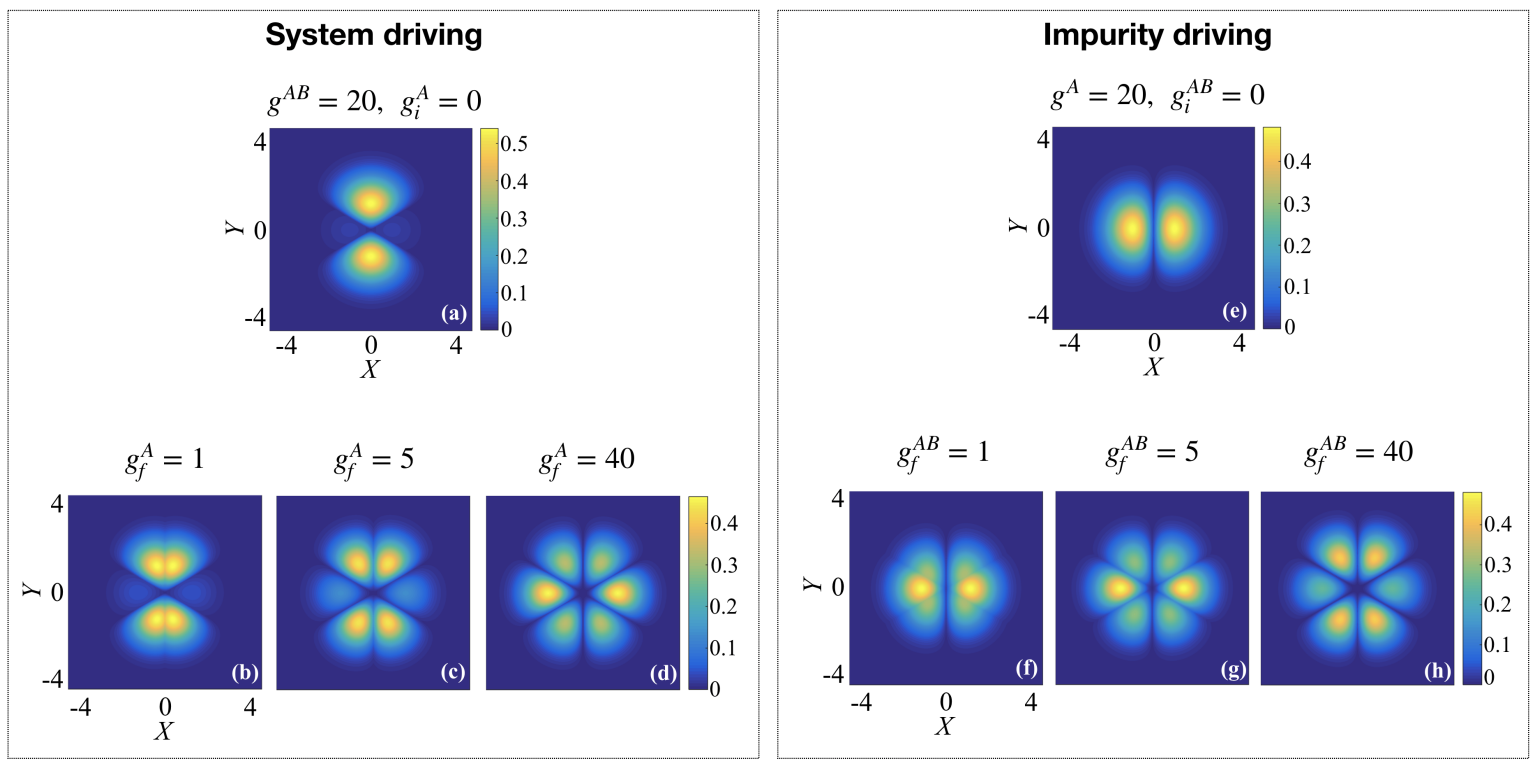

Figure 7. Left panel: driving the interactions of the system when the impurity interaction is fixed at $g^{A B}=20$. (a) Initial state with $g^{A}=0$, (b) target state at $g_{f}^{A}=1,(\mathbf{c}) g_{f}^{A}=5$, and (d) $g_{f}^{A}=40$. Right panel: driving the interaction with the impurity when the system interactions are fixed at $g^{A}=20$. (e) Initial state with $g^{A B}=0$, (f) target state at $g_{f}^{A B}=1$, (g) $g_{f}^{A B}=5$, and (h) $g_{f}^{A B}=40$.

In Figure 8, we show the irreversible work after driving the system interactions (panel (a)) and the impurity interactions (panel (b)). In both cases and for driving to weak final interactions, $g_{f}=1$ or 5 , the STA outperforms the reference and the target state is reached on timescales $t_{f} \approx 10$. This is also confirmed by examining the OBDM at $t_{f}=10$ (see Figure $9 \mathrm{a}, \mathrm{b}$ and $\mathrm{g}$,h), where the states after the STA closely match the target OBDM, while the states after the reference ramp (panels $(\mathrm{d}, \mathrm{e})$ and $(\mathrm{j}, \mathrm{k})$ ) exhibit a slight mismatch at $g^{f}=5$. Indeed, since the strong fixed interactions dominate the structure of the three-particle state, the positions of the particles relative to each other are not significantly altered, which allows for efficient ramping of weak time-dependent interactions. 
(a)

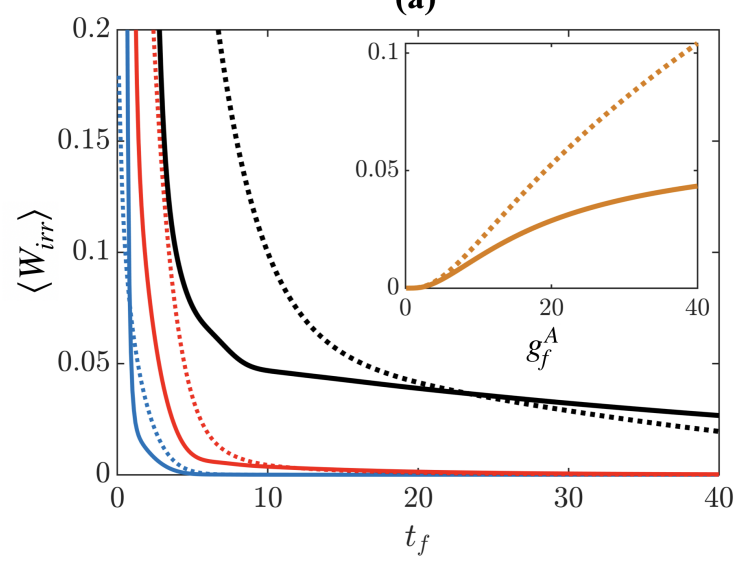

(b)

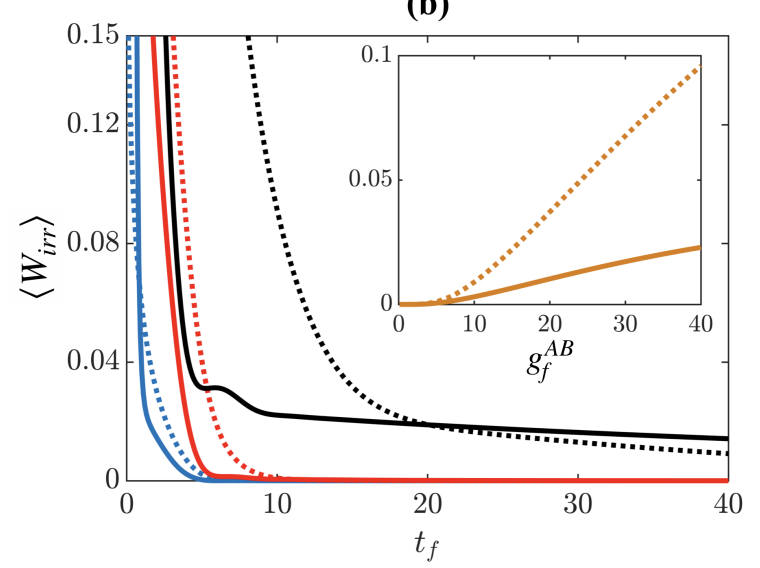

Figure 8. (a) $\left\langle W_{i r r}\right\rangle$ after driving the system interactions in the presence of a strong fixed impurity interaction $g^{A B}=20$. System interactions are driven to $g_{f}^{A}=1$ (blue lines), $g_{f}^{A}=5$ (red lines), and $g_{f}^{A}=40$ (black lines), with the solid lines showing the result of the STA and the dotted lines showing the result of the reference ramp. (b) Impurity driving in the presence of strong fixed system interactions $g^{A}=20$, with final impurity interactions $g_{f}^{A B}=1$ (blue lines), $g_{f}^{A B}=5$ (red lines), and $g_{f}^{A B}=40$ (black lines). Insets show $\left\langle W_{i r r}\right\rangle$ as a function of $g_{f}^{A}$ and $g_{f}^{A B}$, respectively, at $t_{f}=10$.

However, driving to stronger interactions, $g_{f}=40$, in both setups creates large amounts of irreversibility, irregardless of using the STA or the reference (see Figure 8), with the STA producing less $\left\langle W_{i r r}\right\rangle$ for ramp durations $t_{f}<20$. The insets of Figure 8 show $\left\langle W_{i r r}\right\rangle$ at $t_{f}=10$ as a function of the final interaction strengths $g_{f}^{A}$ and $g_{f}^{A B}$, and while the rate of increase for the STA is less than that of the reference, it is still significant. The reason for the failure of the different interaction ramps to reach the target state is the need for particles to spatially reorganize when the intra- and inter-species interactions become comparable (i.e., when $g^{A}(t) \approx g^{A B}$ during system driving and $g^{A B}(t) \approx g^{A}$ during impurity driving). For example, when driving the system interactions, the relative wave-function of the initial state is highly localized along the $X$ direction due to the presence of the strong impurity interactions at $Y= \pm X / \sqrt{3}$ (see Figure 7a), resulting in a phase-separated state in the configuration BAB. Ramping to weak intra-species interactions $\left(g_{f}^{A}=\{1,5\}\right)$ preserves the phase separation as the impurity interaction still dominates the system (see Figure $9 a, b, d, e)$. Increasing the intra-species interaction further to $g^{A}=g^{A B}$ requires the ground state to regain the $C_{6 v}$ symmetry discussed in Section 4; however, this is difficult to achieve dynamically due to the presence of the large fixed inter-species interactions, $g^{A B}=20$, which will suppress tunneling of the density across the delta-function barriers at $Y= \pm X / \sqrt{3}$. In the three particle coordinates, this corresponds to the need for the particles to tunnel through each other [50,51]; however, they cannot reorganize on this short timescale due to the low tunneling rates. Therefore, for the final state of $g_{f}^{A}=40$, the particles will remain in the phase-separated configuration of the initial state, $\mathrm{BAB}$, instead of reaching the configuration of the target state, ABA (see Figure 9, panels (c) and (f)). The same result is observed when driving the impurity interactions (see Figure 9, panels (i) and (1)) but with the opposite configuration of the particles. 

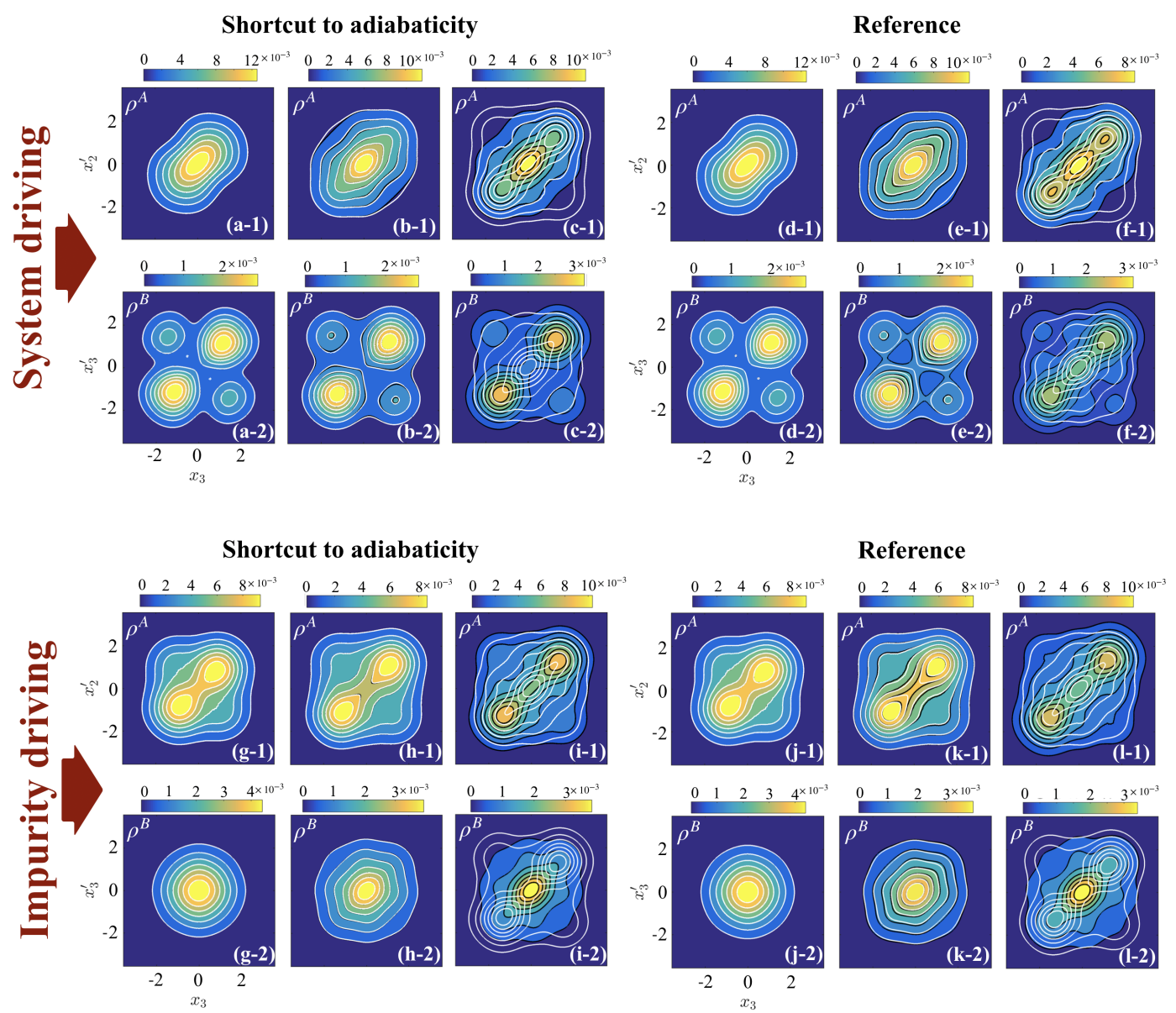

Figure 9. Panels (a-f): Target states (white contour lines) on top of final states (at $t_{f}=10$ ) for driving system interactions between $\mathrm{A}$ atoms in the presence of a fixed impurity interaction $g^{A B}=20$. Panels with index $(-1)$ correspond to $\rho^{A}\left(x_{1}, x_{1}^{\prime}\right)=\rho^{A}\left(x_{2}, x_{2}^{\prime}\right)$, while panels with index $(-2)$ show $\rho^{B}\left(x_{3}, x_{3}^{\prime}\right)$. Panels (a-c) show the STA final states, while panels (d-f) show the final states for the reference pulse, where panels (a) and (d) are for $g_{f}^{A}=1,(\mathbf{b})$ and (e) are for $g_{f}^{A}=5$, and (c) and (f) are for $g_{f}^{A}=40$. Panels (g-1): Target states (white contour lines) on top of final states (at $t_{f}=10$ ) for driving impurity interactions in the presence of a fixed interactions between the A atoms $g^{A}=20$. Panels $(\mathbf{g})$ and $(\mathbf{j})$ are for $g_{f}^{A B}=1,(\mathbf{h})$ and $(\mathbf{k})$ are for $g_{f}^{A B}=5$, and (i) and (l) are for $g_{f}^{A B}=40$.

Since these final states are not eigenstates, their energy exceeds that of the target ground state, resulting in finite $\left\langle W_{i r r}\right\rangle$ (see Figure 8 ) even for long ramp times $\left(t_{f} \approx 40\right.$ ), when we would assume the driving is slow enough to be close to adiabatic. Moreover, one can see that for $t_{f}>20$, the $\left\langle W_{i r r}\right\rangle$ of the reference ramp is lower than that of the STA and that it decreases at a faster rate, suggesting that the higher energy states the reference ramp drives the system through prevent it from being fully trapped in the local energy minimum given by the wrong particle ordering. To understand this in more detail, we show in Figure 10 the average kinetic energy $\langle K\rangle$, trap potential energy $\left\langle V_{\text {trap }}\right\rangle$, and interaction energy $\left\langle V_{\text {int }}\right\rangle$ at the end of the different interaction ramps. One can immediately see that for the STA (black lines), where the ramp is specifically designed to minimize excitations, only small oscillations of the energy components for any $t_{f}$ exist, while in comparison, the reference ramp is not optimized to reduce excitations and therefore large oscillations are present. Furthermore, the kinetic and trap energies, on average, exceed that of the respective target states, whereas the interaction energy is less than that of the target state. This is consistent with the fact that both ramps are unable to achieve the correct ordering of the particles, therefore leaving them in an excited state that is more extended and therefore possesses slightly reduced interaction energies. It is also interesting to note that for long times $\left(t_{f}>20\right)$, the reference ramp gives slightly more correct energies, which again is due to it 
exciting higher lying states that help parts of the wave-function to not be trapped in the wrong state and encourages inter-particle tunneling.
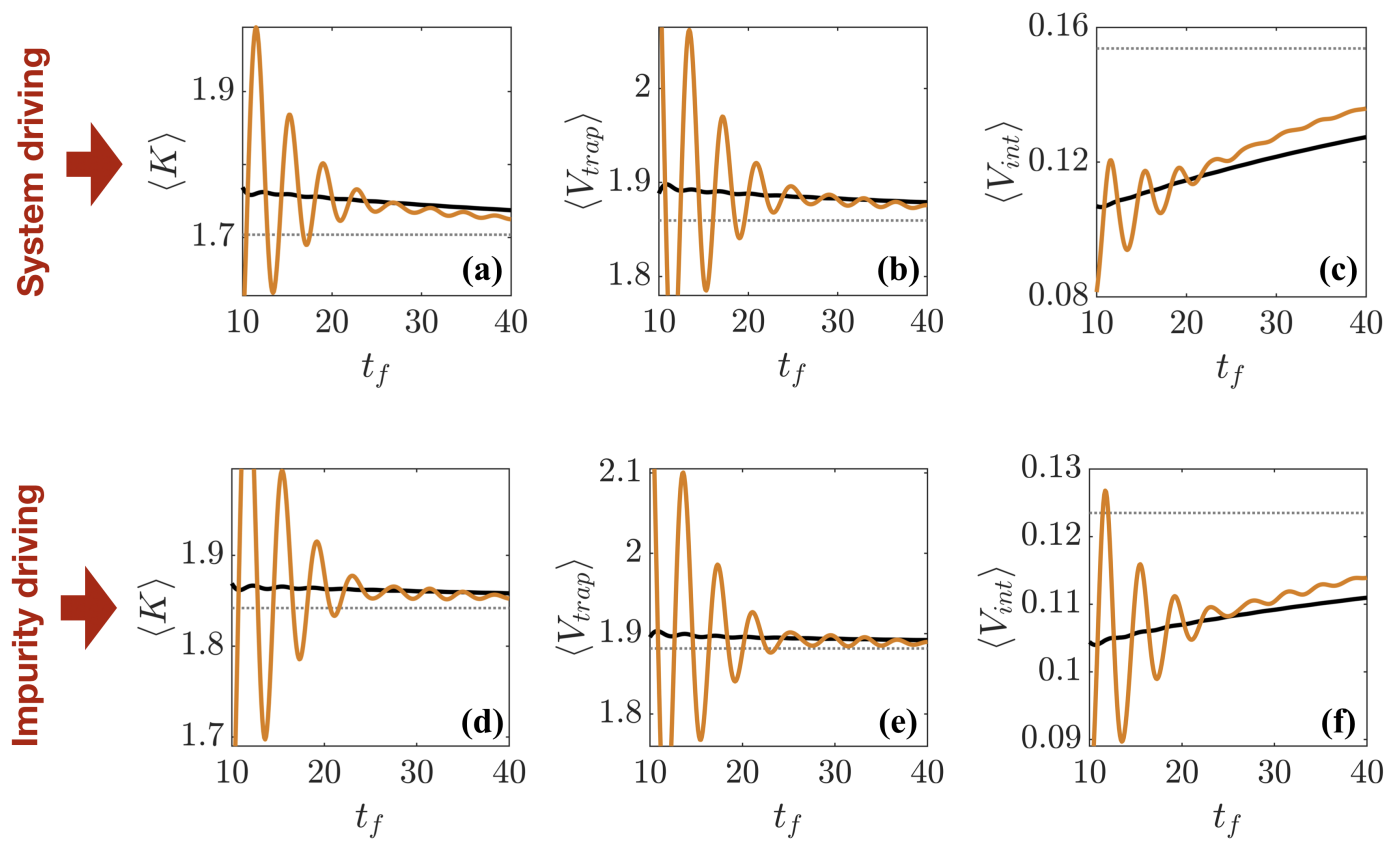

Figure 10. (a,d) Kinetic energy, (b,e) potential trap energy, and $(\mathbf{c}, \mathbf{f})$ interaction energy as a function of $t_{f}$ after using the STA (black solid line) and reference (orange solid line), with the adiabatic energies shown as the thin dotted line. $(\mathbf{a}-\mathbf{c})$ show the result of driving the system to $g_{f}^{A}=40$, while $(\mathbf{d}-\mathbf{f})$ show the result of driving the impurity to $g_{f}^{A B}=40$. Note the different scales on the subplots.

\section{Conclusions \& Outlook}

We have presented a study that extends the use of shortcuts to adiabaticity to interacting few-particle systems. Using a variational technique, we have shown that certain limits exist in which effective shortcuts for these systems, which can possess non-trivial ground states, can be designed. Our approach works well for a number of cases, such as for three identical particles and in the presence of fixed weak interactions, and consistently outperforms a non-optimized reference ramp. The timescales for which high-fidelity states can be reached are comparable to those achievable using optimal control techniques in related few-body systems [6]. We have also shown that the approach fails when the system is required to go through a phase-separation transition that requires the particles to tunnel through each other to achieve a new spatial ordering. As the STA is designed to reduce spurious excitations, the particles become trapped in a quasi-stationary state with a lifetime much longer than the driving process. One way to enhance the tunneling rates of the particles during the interaction ramp would be to simultaneously modulate the trap potential [52], apply periodic shaking of the trap [53], or heat the system to allow for thermal hopping [54]. These avenues will be explored in future work. In addition, while our superposition ansatz is effective for weak interactions (see Appendix A), it can be improved to more accurately describe the phase-separated regime by introducing intermediate states. Moreover, while our approach has been shown to work successfully when driving interactions in the mean-field regime $[19,20]$, it would be interesting to extend these techniques to the full many-body state, where the orthogonality catastrophe will have a significant effect on the dynamics [10,55-57].

Author Contributions: This project was conceptualized by T.F., J.L. and T.B.; software, validation, data curation, and formal analysis was by A.K.; writing, review and editing was by all authors.

Funding: This work was supported by the Okinawa Institute of Science and Technology Graduate University. TF acknowledges funding support under JSPS KAKENHI-18K13507. 
Acknowledgments: We acknowledge support from the Scientific Computing and Data Analysis section at the Okinawa Institute of Science and Technology Graduate University. AK acknowledges support and infinite patience of Cecilia Cormick.

Conflicts of Interest: The authors declare no conflict of interest.

\section{Appendix A. Accuracy of the Ansatz}

To quantify the effectiveness of the interpolatory ansatz we use to design the STA ramps, we compare it with the exact eigenstates of the interacting Hamiltonian. In Figure A1, we show the fidelity as a function of the interaction strength, $\left|\left\langle\varphi(g) \mid \psi_{\text {exact }}(g)\right\rangle\right|^{2}$, where $\varphi(g)$ is the ansatz from (11) and $\psi_{\text {exact }}(g)$ is the instantaneous eigenstate obtained through exact diagonalization.
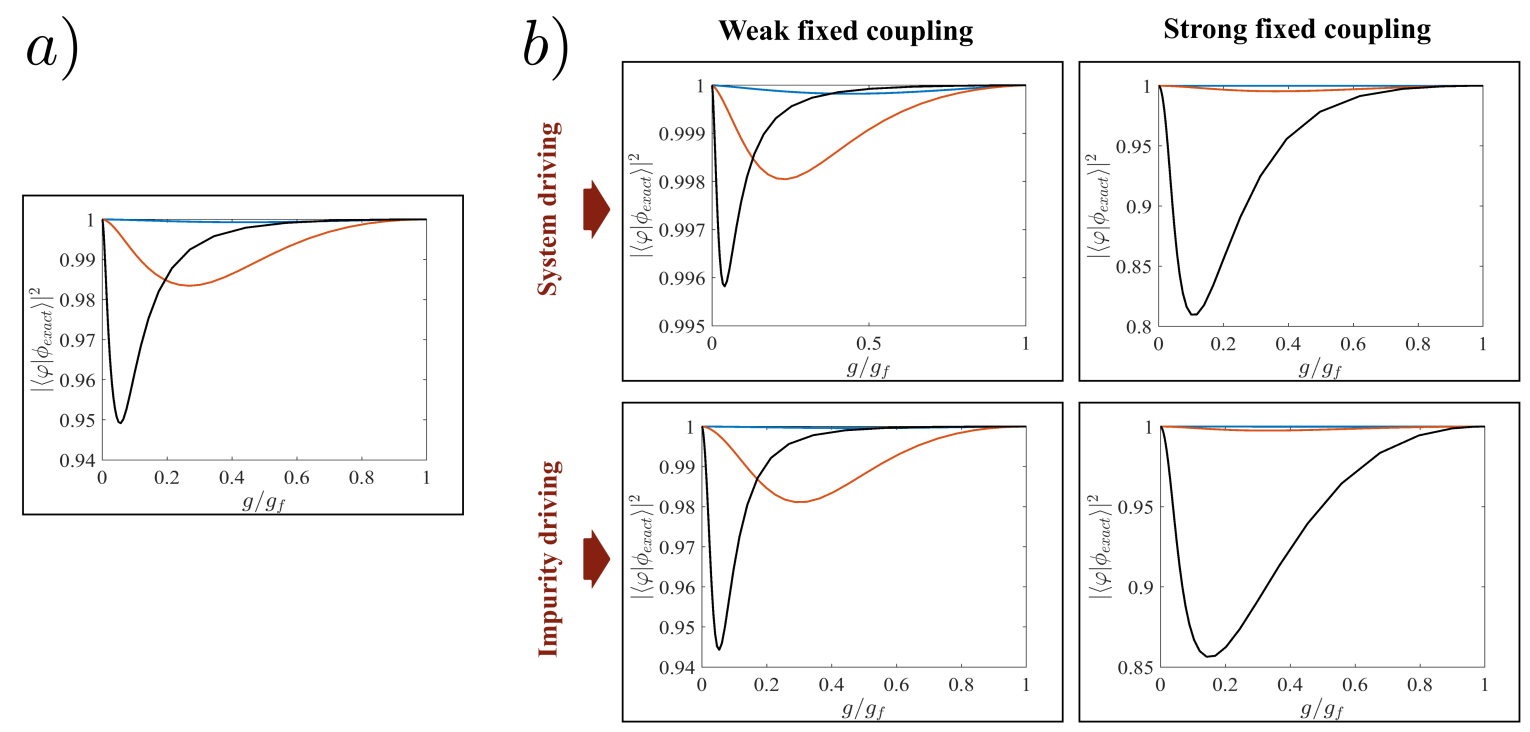

Figure A1. Fidelity between instantaneous ground state obtained with exact diagonalization of the Hamiltonian and the interpolatory ansatz as a function of $g / g_{f}$. Panel (a) is the fidelity for three identical particles, while panel (b) shows the fidelity for system and impurity driving. The final interactions chosen are $g_{f}=1$ (blue lines), $g_{f}=5$ (red lines), and $g_{f}=40$ (black lines).

In all cases when the final interaction strength is weak $\left(g_{f}=\{1,5\}\right)$, the ansatz matches the exact eigenstate with almost unit fidelity. However, for strong fixed couplings and final interactions $g_{f}=40$, the fidelity drops significantly during the beginning of the interaction ramp as the ansatz fails to accurately account for the competition between the different interaction strengths. Similar deficiencies have been noted using other variational ansatzes for interacting few-body systems [39], and this can have a detrimental effect on the success of the STA in the presence of phase separation.

\section{References}

1. Lukin, M.D. Colloquium: Trapping and manipulating photon states in atomic ensembles. Rev. Mod. Phys. 2003, 75, 457-472, doi:10.1103/RevModPhys.75.457. [CrossRef]

2. Thompson, J.D.; Tiecke, T.G.; Zibrov, A.S.; Vuletić, V.; Lukin, M.D. Coherence and Raman Sideband Cooling of a Single Atom in an Optical Tweezer. Phys. Rev. Lett. 2013, 110, 133001, doi:10.1103/PhysRevLett.110.133001. [CrossRef] [PubMed]

3. Levine, H.; Keesling, A.; Omran, A.; Bernien, H.; Schwartz, S.; Zibrov, A.S.; Endres, M.; Greiner, M.; Vuletić, V.; Lukin, M.D. High-Fidelity Control and Entanglement of Rydberg-Atom Qubits. Phys. Rev. Lett. 2018, 121, 123603, doi:10.1103/PhysRevLett.121.123603. [CrossRef] [PubMed]

4. Werschnik, J.; Gross, E.K.U. Quantum optimal control theory. J. Phys. B: At. Mol. Opt. Phys. 2007, 40, R175-R211, doi:10.1088/0953-4075/40/18/r01. [CrossRef] 
5. Van Frank, S.; Bonneau, M.; Schmiedmayer, J.; Hild, S.; Gross, C.; Cheneau, M.; Bloch, I.; Pichler, T.; Negretti, A.; Calarco, T.; et al. Optimal control of complex atomic quantum systems. Sci. Rep. 2016, 6, 34187. [CrossRef]

6. Li, X.; Pęcak, D.; Sowiński, T.; Sherson, J.; Nielsen, A.E.B. Global optimization for quantum dynamics of few-fermion systems. Phys. Rev. A 2018, 97, 033602, doi:10.1103/PhysRevA.97.033602. [CrossRef]

7. Torrontegui, E.; Ibáñez, S.; Martínez-Garaot, S.; Modugno, M.; del Campo, A.; Guéry-Odelin, D.; Ruschhaupt, A.; Chen, X.; Muga, J.G. Chapter 2-Shortcuts to Adiabaticity. In Advances in Atomic, Molecular, and Optical Physics; Arimondo, E., Berman, P.R., Lin, C.C., Eds.; Academic Press: Cambridge, MA, USA, 2013; Volume 62, pp. 117-169.

8. Del Campo, A. Shortcuts to Adiabaticity by Counterdiabatic Driving. Phys. Rev. Lett. 2013, 111, 100502, doi:10.1103/PhysRevLett.111.100502. [CrossRef]

9. Cui, Y.Y.; Chen, X.; Muga, J.G. Transient Particle Energies in Shortcuts to Adiabatic Expansions of Harmonic Traps. J. Phys. Chem. A 2016, 120, 2962-2969. [CrossRef]

10. Sels, D.; Polkovnikov, A. Minimizing irreversible losses in quantum systems by local counterdiabatic driving. Proc. Natl. Acad. Sci. USA 2017, 114, E3909-E3916. [CrossRef]

11. Guéry-Odelin, D.; Ruschhaupt, A.; Kiely, A.; Torrontegui, E.; Martínez-Garaot, S.; Muga, J.G. Shortcuts to adiabaticity: Concepts, methods, and applications. arXiv 2019, arXiv:1904.08448.

12. Schaff, J.F.; Song, X.L.; Vignolo, P.; Labeyrie, G. Fast optimal transition between two equilibrium states. Phys. Rev. A 2010, 82, 033430. [CrossRef]

13. Schaff, J.F.; Song, X.L.; Capuzzi, P.; Vignolo, P.; Labeyrie, G. Shortcut to adiabaticity for an interacting Bose-Einstein condensate. Europhys. Lett. 2011, 93, 23001. [CrossRef]

14. Schaff, J.F.; Capuzzi, P.; Labeyrie, G.; Vignolo, P. Shortcuts to adiabaticity for trapped ultracold gases. New J. Phys. 2011, 13, 113017. [CrossRef]

15. Rohringer, W.; Fischer, D.; Steiner, F.; Mazets, I.E.; Schmiedmayer, J.; Trupke, M. Non-equilibrium scale invariance and shortcuts to adiabaticity in a one-dimensional Bose gas. Sci. Rep. 2015, 5, 9820. [CrossRef]

16. Deng, S.; Diao, P.; Yu, Q.; del Campo, A.; Wu, H. Shortcuts to adiabaticity in the strongly coupled regime: Nonadiabatic control of a unitary Fermi gas. Phys. Rev. A 2018, 97, 013628, doi:10.1103/PhysRevA.97.013628. [CrossRef]

17. Diao, P.; Deng, S.; Li, F.; Yu, S.; Chenu, A.; del Campo, A.; Wu, H. Shortcuts to adiabaticity in Fermi gases. New J. Phys. 2018, 20, 105004, doi:10.1088/1367-2630/aae45e. [CrossRef]

18. Pérez-García, V.M.; Michinel, H.; Cirac, J.I.; Lewenstein, M.; Zoller, P. Low Energy Excitations of a Bose-Einstein Condensate: A Time-Dependent Variational Analysis. Phys. Rev. Lett. 1996, 77, 5320-5323, doi:10.1103/PhysRevLett.77.5320. [CrossRef]

19. Li, J.; Sun, K.; Chen, X. Shortcut to adiabatic control of soliton matter waves by tunable interaction. Sci. Rep. 2016, 6, 38258. [CrossRef]

20. Li, J.; Fogarty, T.; Campbell, S.; Chen, X.; Busch, T. An efficient nonlinear Feshbach engine. New J. Phys. 2018, 20, 015005, doi:10.1088/1367-2630/aa9cd8. [CrossRef]

21. Fogarty, T.; Ruks, L.; Li, J.; Busch, T. Fast control of interactions in an ultracold two atom system: Managing correlations and irreversibility. SciPost Phys. 2019, 6, 21, doi:10.21468/SciPostPhys.6.2.021. [CrossRef]

22. Hall, D.S.; Matthews, M.R.; Ensher, J.R.; Wieman, C.E.; Cornell, E.A. Dynamics of Component Separation in a Binary Mixture of Bose-Einstein Condensates. Phys. Rev. Lett. 1998, 81, 1539-1542, doi:10.1103/ PhysRevLett.81.1539. [CrossRef]

23. Mishra, T.; Pai, R.V.; Das, B.P. Phase separation in a two-species Bose mixture. Phys. Rev. A 2007, 76, 013604, doi:10.1103/PhysRevA.76.013604. [CrossRef]

24. García-March, M.A.; Juliá-Díaz, B.; Astrakharchik, G.E.; Busch, T.; Boronat, J.; Polls, A. Quantum correlations and spatial localization in one-dimensional ultracold bosonic mixtures. New J. Phys. 2014, 16, 103004, doi:10.1088/1367-2630/16/10/103004. [CrossRef]

25. Lee, K.L.; Jørgensen, N.B.; Liu, I.K.; Wacker, L.; Arlt, J.J.; Proukakis, N.P. Phase separation and dynamics of two-component Bose-Einstein condensates. Phys. Rev. A 2016, 94, 013602, doi:10.1103/PhysRevA.94.013602. [CrossRef]

26. Köhler, T.; Góral, K.; Julienne, P.S. Production of cold molecules via magnetically tunable Feshbach resonances. Rev. Mod. Phys. 2006, 78, 1311-1361, doi:10.1103/RevModPhys.78.1311. [CrossRef] 
27. Chin, C.; Grimm, R.; Julienne, P.; Tiesinga, E. Feshbach resonances in ultracold gases. Rev. Mod. Phys. 2010, 82, 1225-1286, doi:10.1103/RevModPhys.82.1225. [CrossRef]

28. Olshanii, M. Atomic Scattering in the Presence of an External Confinement and a Gas of Impenetrable Bosons. Phys. Rev. Lett. 1998, 81, 938-941, doi:10.1103/PhysRevLett.81.938. [CrossRef]

29. Zinner, N.T.; Volosniev, A.G.; Fedorov, D.V.; Jensen, A.S.; Valiente, M. Fractional energy states of strongly interacting bosons in one dimension. EPL (Europhys. Lett.) 2014, 107, 60003, doi:10.1209/0295-5075/107/60003. [CrossRef]

30. Zinner, N.T. Strongly interacting mesoscopic systems of anyons in one dimension. Phys. Rev. A 2015, 92, 063634, doi:10.1103/PhysRevA.92.063634. [CrossRef]

31. García-March, M.A.; Juliá-Díaz, B.; Astrakharchik, G.E.; Boronat, J.; Polls, A. Distinguishability, degeneracy, and correlations in three harmonically trapped bosons in one dimension. Phys. Rev. A 2014, 90, 063605, doi:10.1103/PhysRevA.90.063605. [CrossRef]

32. Garcia-March, M.A.; Juliá-Díaz, B.; Astrakharchik, G.E.; Busch, T.; Boronat, J.; Polls, A. Sharp crossover from composite fermionization to phase separation in microscopic mixtures of ultracold bosons. Phys. Rev. A 2013, 88, 063604, doi:10.1103/PhysRevA.88.063604. [CrossRef]

33. Cazalilla, M.A.; Ho, A.F. Instabilities in Binary Mixtures of One-Dimensional Quantum Degenerate Gases. Phys. Rev. Lett. 2003, 91, 150403, doi:10.1103/PhysRevLett.91.150403. [CrossRef] [PubMed]

34. Alon, O.E.; Streltsov, A.I.; Cederbaum, L.S. Demixing of Bosonic Mixtures in Optical Lattices from Macroscopic to Microscopic Scales. Phys. Rev. Lett. 2006, 97, 230403, doi:10.1103/PhysRevLett.97.230403. [CrossRef] [PubMed]

35. Zöllner, S.; Meyer, H.D.; Schmelcher, P. Composite fermionization of one-dimensional Bose-Bose mixtures. Phys. Rev. A 2008, 78, 013629, doi:10.1103/PhysRevA.78.013629. [CrossRef]

36. García-March, M.Á.; Fogarty, T.; Campbell, S.; Busch, T.; Paternostro, M. Non-equilibrium thermodynamics of harmonically trapped bosons. New J. Phys. 2016, 18, 103035, doi:10.1088/1367-2630/18/10/103035. [CrossRef]

37. Deffner, S.; Jarzynski, C.; del Campo, A. Classical and Quantum Shortcuts to Adiabaticity for Scale-Invariant Driving. Phys. Rev. X 2014, 4, 021013, doi:10.1103/PhysRevX.4.021013. [CrossRef]

38. Andersen, M.E.S.; Dehkharghani, A.S.; Volosniev, A.G.; Lindgren, E.J.; Zinner, N.T. An interpolatory ansatz captures the physics of one-dimensional confined Fermi systems. Sci. Rep. 2016, 6, 28362. [CrossRef]

39. Pęcak, D.; Dehkharghani, A.S.; Zinner, N.T.; Sowiński, T. Four fermions in a one-dimensional harmonic trap: Accuracy of a variational-ansatz approach. Phys. Rev. A 2017, 95, 053632, doi:10.1103/PhysRevA.95.053632. [CrossRef]

40. Harshman, N.L. Symmetries of three harmonically trapped particles in one dimension. Phys. Rev. A 2012, 86, 052122, doi:10.1103/PhysRevA.86.052122. [CrossRef]

41. Harshman, N.L. One-Dimensional Traps, Two-Body Interactions, Few-Body Symmetries: I. One, Two, and Three Particles. Few-Body Sys. 2016, 57, 11-43, doi:10.1007/s00601-015-1024-6. [CrossRef]

42. Kinoshita, T.; Wenger, T.; Weiss, D.S. Observation of a One-Dimensional Tonks-Girardeau Gas. Science 2004, 305, 1125-1128. [CrossRef] [PubMed]

43. Minguzzi, A.; Gangardt, D.M. Exact Coherent States of a Harmonically Confined Tonks-Girardeau Gas. Phys. Rev. Lett. 2005, 94, 240404, doi:10.1103/PhysRevLett.94.240404. [CrossRef]

44. Haller, E.; Gustavsson, M.; Mark, M.J.; Danzl, J.G.; Hart, R.; Pupillo, G.; Nägerl, H.C. Realization of an Excited, Strongly Correlated Quantum Gas Phase. Science 2009, 325, 1224-1227. [CrossRef] [PubMed]

45. Kormos, M.; Collura, M.; Calabrese, P. Analytic results for a quantum quench from free to hard-core one-dimensional bosons. Phys. Rev. A 2014, 89, 013609, doi:10.1103/PhysRevA.89.013609. [CrossRef]

46. Kehrberger, L.M.A.; Bolsinger, V.J.; Schmelcher, P. Quantum dynamics of two trapped bosons following infinite interaction quenches. Phys. Rev. A 2018, 97, 013606, doi:10.1103/PhysRevA.97.013606. [CrossRef]

47. Nicklas, E.; Karl, M.; Höfer, M.; Johnson, A.; Muessel, W.; Strobel, H.; Tomkovič, J.; Gasenzer, T.; Oberthaler, M.K. Observation of scaling in the dynamics of a strongly quenched quantum gas. Phys. Rev. Lett. 2015, 115, 245301. [CrossRef]

48. Bisset, R.; Wilson, R.; Ticknor, C. Scaling of fluctuations in a trapped binary condensate. Phys. Rev. A 2015, 91, 053613. [CrossRef] 
49. Mistakidis, S.I.; Katsimiga, G.C.; Kevrekidis, P.G.; Schmelcher, P. Correlation effects in the quench-induced phase separation dynamics of a two species ultracold quantum gas. New J. Phys. 2018, 20, 043052, doi:10.1088/ 1367-2630/aabc6a. [CrossRef]

50. Pflanzer, A.C.; Zöllner, S.; Schmelcher, P. Material-barrier tunnelling in one-dimensional few-boson mixtures. J. Phys. B At. Mol. Opt. Phys. 2009, 42, 231002, doi:10.1088/0953-4075/42/23/231002. [CrossRef]

51. Pflanzer, A.C.; Zöllner, S.; Schmelcher, P. Interspecies tunneling in one-dimensional Bose mixtures. Phys. Rev. A 2010, 81, 023612, doi:10.1103/PhysRevA.81.023612. [CrossRef]

52. Martínez-Garaot, S.; Palmero, M.; Muga, J.G.; Guéry-Odelin, D. Fast driving between arbitrary states of a quantum particle by trap deformation. Phys. Rev. A 2016, 94, 063418, doi:10.1103/PhysRevA.94.063418. [CrossRef]

53. Zenesini, A.; Lignier, H.; Sias, C.; Morsch, O.; Ciampini, D.; Arimondo, E. Tunneling control and localization for Bose-Einstein condensates in a frequency modulated optical lattice. Laser Phys. 2010, 20, 1182-1189, doi:10.1134/S1054660X10090410. [CrossRef]

54. Wu, J.H.; Qi, R.; Ji, A.C.; Liu, W.M. Quantum tunneling of ultracold atoms in optical traps. Front. Phys. 2014, 9, 137-152, doi:10.1007/s11467-013-0359-z. [CrossRef]

55. Goold, J.; Fogarty, T.; Lo Gullo, N.; Paternostro, M.; Busch, T. Orthogonality catastrophe as a consequence of qubit embedding in an ultracold Fermi gas. Phys. Rev. A 2011, 84, 063632, doi:10.1103/PhysRevA.84.063632. [CrossRef]

56. Knap, M.; Shashi, A.; Nishida, Y.; Imambekov, A.; Abanin, D.A.; Demler, E. Time-Dependent Impurity in Ultracold Fermions: Orthogonality Catastrophe and Beyond. Phys. Rev. X 2012, 2, 041020, doi:10.1103/ PhysRevX.2.041020. [CrossRef]

57. Campbell, S.; García-March, M.A.; Fogarty, T.; Busch, T. Quenching small quantum gases: Genesis of the orthogonality catastrophe. Phys. Rev. A 2014, 90, 013617, doi:10.1103/PhysRevA.90.013617. [CrossRef]

(C) 2019 by the authors. Licensee MDPI, Basel, Switzerland. This article is an open access article distributed under the terms and conditions of the Creative Commons Attribution (CC BY) license (http:/ / creativecommons.org/licenses/by/4.0/). 